\title{
Workplace wellbeing groups and champions: a remedy for lack of human resource for workplace wellbeing functions
}

\author{
Enoka P Wickramasinghe \\ The Directorate of Mental Health, Ministry of Health, Sri Lanka \\ *Correspondence: enoka7@gmail.com (iD https://orcid.org/0000-0003-2129-5496 \\ DOI: https://doi.org/10.4038/jccpsl.v26i1.8270
}

Received on 02 February 2020

Accepted on 07 March 2020

\section{Introduction}

Globally, employee wellbeing has gained much interest and attention among policy makers and employers due to its impact on employee performance and organizational effectiveness. Hence, work settings are identified as venues for investing on employee wellbeing, disease prevention and health promotion. Wellbeing is an overarching term which encompasses both health and wellness and is characterised not only by good health or wellness but also comfort, satisfaction with one's work and home life, personal prosperity and some measure of happiness. Therefore, wellbeing of an employee represents positive functioning of the worker rather than traditional focus on absence of ill-health and poor functioning only (1). A major challenge faced by the governments in providing occupational health and safety (OHS) services for the workforce is lack of service personnel to attend to the range of health needs in work settings. Establishment of workplace wellbeing groups and champions initiative has shown success as a sustainable and feasible solution to face this challenge (1).

Nearly half of the total global population consists of workers (3.49 billion), of which majority live in the low- and middle-income countries (LMIC) and middle-income countries (MIC). In Sri Lanka, out of the 21.7 million population, 8.64 million are workers (2-3). Moreover, many employees spend majority of their waking hours in workplaces or are engaged in work (4). Hence, focusing on employee wellbeing would firstly lead to reductions in healthcare costs from accidents and injuries, care provision, mental health issues (4) and health insurance premiums, and secondly increase employee engagement, working capacity and productivity and reduce sickness absenteeism and staff turnover. The cumulative effect of all these would facilitate organizational effectiveness and economy of the country in the long run. Also, evidence suggests the organizations where employee health and wellbeing are addressed have shown to outperform those that do not by $10 \%$ (5). Hence, greater return on investment through employee wellbeing programs have been demonstrated (5). As a result, most enterprises and organizations particularly in developed countries and large organizations in the corporate sector focus on implementing employee wellbeing programs in workplaces (1).

On the other hand, in achieving Sustainable Development Goals (SDG), governments are committed to restore health and safety of the workforce to ensure decent work (SDG 8) and health and wellbeing (SDG 3) 
for all in a country (6). Adding to these, the economic and social costs due to the unacceptable levels of workrelated stress and mental health problems draw urgent attention of the government on promoting worker wellbeing. However, a challenge most countries face in carrying out health and safety functions and workplace wellbeing programs is lack of human resource for carrying out such interventions at workplaces. According to the report on situational analysis of OHS in Sri Lanka 2016, lack of health and safety inspectors in divisional level for OHS was a major drawback in carrying out OH\&S functions in the country (7). Employers also have a shared responsibility in initiating and continuing workplace wellbeing programs relevant to specific work settings for the benefit of the employees and thereby the organization. Organizational level initiation of such interventions would thereby provide ownership to the organization and facilitate sustainability, and also reduce the burden on the government too.

Workplace wellness programs have traditionally been focusing on individual behaviour change. However, research has revealed that organizational culture specific factors may be key determinants of the effectiveness of organization-wide interventions focusing on mental health and wellbeing. Hence addressing unique organizational needs through creation of a 'healthy organization culture' and adopting a participatory and non-stigmatizing approach are identified as effective systemic methods (8). It is thus essential to acknowledge the full picture of the workplace environment and impact of the employee experience by putting employees at the centre. This necessitates redesigning workplace practices particularly those of wellbeing to fit with employee interests and needs through participatory approaches. In this effort management involvement and commitment throughout is equally essential. Most notably addressing the obstacles and creating conditions for employees to practice healthy lifestyles within work settings are important (9).

Pioneering this task under the Helping Great Britain Work Well Strategy, the Health and Safety Executive of United Kingdom (UK) has introduced Wellbeing Champion Program (WCP) in workplaces as a novel approach to implement employee wellbeing programs in the UK (8-9). The wellbeing or business champions are identified as drivers to forward organizational change with the aim of producing a culture that promotes wellbeing specific to the respective work setting.

\section{Workplace wellbeing champions}

Wellbeing champion is a member of staff employed by an organization who is supported to design, deliver and perhaps evaluate healthy workplace programmes (8). The champion is a self-identified employee who value health and wellbeing, has a genuine interest in supporting their colleagues and want to promote a 'grassroots' approach to wellness programs. Hence, the crux of the role of champion is to regularly engage and empower the colleagues in activities that promote their own wellbeing.

\section{Wellbeing champion program (WCP)}

This is a multi-stage process (10). Identification and training of dedicated wellbeing champions is the key to success for this organization driven wellbeing program. The number of champions invited from each workplace depends on the size of the organization and the existence of varying units within the work setting. The key stages of the WCP are:

- Program development

- Resource development (e.g. identification of employee wellbeing specialists; development of training manual, information education communication material and communication messages)

- Recruitment of the champions

- Orientation training for the wellbeing champions

- Regular evaluation

\section{Training of wellbeing champions}

The employee wellbeing specialists from either Health and Safety Executive staff or Local Authority staff, deliver workshops for the current and new wellbeing champions to connect with available resources, offer creative and feasible methods to implement ideas (10). In addition, wellbeing champions are provided with activities and tools for them to create meaningful activities within their work groups. 
However, champions are empowered with skills to develop new tools and practices along with their work members themselves, because ownership and trust are most important characteristics of program adherence.

Champions are given the autonomy to promote programs of personal and work group interest. The promotions can cover several domains of wellbeing such as physical activity, healthy diet, volunteerism, team building, social interaction, stress management, financial wellbeing and new experiences of the group. Hence, the capacity building emphasises skill development in these aspects.

\section{Role design of the champion}

The functions of these wellbeing champions were based on an organizational wellbeing strategy, which mainly focused on six pillars of wellbeing: social, physical, emotional, financial, work-life integration and meaning of work. Behaviour change principles and social cognitive theory provide base for a well-designed WCP, emphasising the importance of peer support and social networks for individual behaviour (9). Wellbeing champions provide a critical peer support needed to improve and maintain healthy behaviours among coworkers.

The wellbeing team is expected to create diverse, scalable, expert driven evidence-based programs to enhance employee wellbeing. An agreement with organizational leadership is obtained to provide at least 1-5 hours per month for champions to carry out activities and attend meetings during workdays. These expectations are reviewed through a supervisor agreement form. Supervisors are identified within the work setting for the champions. Clear roles and responsibilities between the supervisor and the wellbeing champions are established at the onset of the champion taking the role (9).

\section{Recognition and retention of champions}

Retention, recognition and evaluation of champions determine the effectiveness of this program and associated activities in sustaining it. As a measure of rewarding the champions and their supervisors, the wellbeing specialists coordinate annual recognition banquet, celebrate both champion role and the support of the supervisor. Awards are given for honours such as 'supervisor of the year', 'well-being champion of the year', 'best well-space' and 'unsung hero' (9).

\section{Effectiveness of wellbeing champions in promoting workplace wellbeing}

In a study carried out among 65,000 employees at a geographically and functionally diverse health care organization (1), with a 73\% responsive rate; revealed presence of a wellbeing champion was significantly associated with a more favourable impression of personal and organizational wellbeing.

Combined systemic approaches working at both organizational and individual level to foster healthy organizations and sustain individual wellbeing worked particularly well if they were also participatory and followed a bottom-up approach (8).

In a forbidding economic climate, sustaining change should not rest only on a charismatic champion, but involve developing high-quality models for capacity building and embedding the practice into the system or organizational culture. Therefore, the effectiveness and sustainability will depend on employee engagement, identification of organizational culture and exploration of strengths, weaknesses, opportunities and threats within the organization for such programs (11). Public Health England in its Complete Handbook for Champions at Workplace gives five key functional areas for workplace champions (11).

\section{Five ways to wellbeing at work}

- Connect Talk \& Listen, be there, Feel connected

- Give Your time, words and presence

- Take Notice Remember the simple things that give you joy

- Keep Learning Embrace new experiences, See opportunities, Surprise yourself

- Be Active Do what you can, enjoy what you do, Move your mood 
Wellbeing Champion Programs have been proposed as a high reach strategy to enhance employee participation in broader wellness at workplace and has an impact on workplace healthy living behaviours (12). However, WCP could impact the wellbeing and satisfaction when implemented as part of a comprehensive employee wellbeing strategy. Furthermore, leadership influence is likely to be pivotal hence engaging senior management and employers in promoting activities from inception is vital (8-9).

\section{Conclusion}

Implementation of well-coordinated, employee centred, and organization specific structured workplace wellbeing champion programs have found to be effective in promoting personal and organizational wellbeing. In the context of lack of human resource for workplace health and wellbeing functions in Sri Lanka, possibility of incorporation of this type of evidence based effective interventions may pave path for solutions for a long-standing issue which existed in the occupational health and safety system in the country.

\section{References}

1. Pruyne E. Corporate investment in employee wellbeing, the emerging strategic imperative. Ashridge Business School and Nuffield Health Surrey: Nuffield Health, 2011.

2. ILO. World Employment Social Outlook Trends 2019. Geneva: International Labour Office, 2019.

3. IBRD-IDA. Labour Force. World Bank, 2019. Available from: https://data.worldbank.org/indicator/ SL.TLF.TOTL.IN.
4. Baicker K, David Cutler D, Song Z. Workplace wellness programs can generate savings. Health Affairs 2010; 29(2): 304-311.

5. Aston L. Helping workers help themselves. Occupational Health 2010; 62(11): 29-32.

6. United Nations. Report of the Inter-Agency and Expert Group on Sustainable Development Goal Indicators (E/CN.3/2016/2/Rev.1). Geneva: Economic and Social Council, 15 December 2016.

7. Department of Community Medicine. Report on the Situation Analysis on Occupational Health \& Safety in Sri Lanka. Colombo: WHO collaborating centre for Training \& Research in Occupational Health, Faculty of Medicine, University of Colombo, 2016.

8. Robinson M, Tilford S, Branney P, Kinsella K. Championing mental health at work: emerging practice from innovative projects in the UK. Health Promotion International 2013; 29(3): 583-595.

9. Wieneke KC, Egginton JS, Jenkins SM, Kruse GC, Jimenez FL, Mungo MM, Riley BA, Limburg PJ. Wellbeing champion impact on employee engagement, staff satisfaction, and employee well-being. Mayo Clinic Proceedings. Innovations, Quality and Outcomes 2019; 3(2): 106-115.

10. Health \& Safety at Work. Summary statistics for Great Britain 2017. UK: Health \& Safety Executive, 2017.

Available from: https://www.hse.gov.uk/statistics/ overall/hssh1718.pdf

11. Department of Health. A Complete Handbook for Champions in the Workplace. UK: Public Health England.

Available from: https://www.time-to-change.org.uk/ sites/default/files/FINAL/handbook.pdf.

12. Mental Health Commission. Thrive at work wellbeing commitment. Growing healthier and happier workplaces. West Midlands: Department of Health. Available from: www.thrive-at-work-commitmentframework.pdf. 